Utah State University

DigitalCommons@USU

Mathematics and Statistics Faculty

Presentations

Mathematics and Statistics

2016

\title{
Population density, not host competence, drives patterns of disease in an invaded community
}

C. L. Searle

Follow this and additional works at: https://digitalcommons.usu.edu/mathsci_presentations

Part of the Mathematics Commons

\section{Recommended Citation}

Searle, C. L., "Population density, not host competence, drives patterns of disease in an invaded community" (2016). Mathematics and Statistics Faculty Presentations. Paper 56.

https://digitalcommons.usu.edu/mathsci_presentations/56

This Article is brought to you for free and open access by the Mathematics and Statistics at DigitalCommons@USU. It has been accepted for inclusion in Mathematics and Statistics Faculty Presentations by an authorized administrator of DigitalCommons@USU. For more information, please contact digitalcommons@usu.edu.

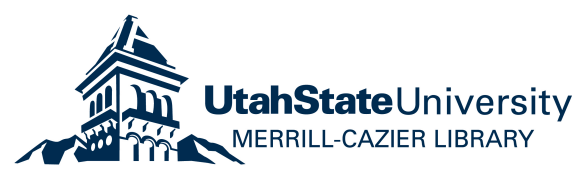




\title{
Population Density, Not Host Competence, Drives Patterns of Disease in an Invaded Community
}

\author{
Catherine L. Searle, ${ }^{1,2, \star}$ Michael H. Cortez, ${ }^{3}$ Katherine K. Hunsberger, ${ }^{2}$ Dylan C. Grippi, ${ }^{2}$ \\ Isabella A. Oleksy, ${ }^{2,4}$ Clara L. Shaw, ${ }^{2}$ Solanus B. de la Serna, ${ }^{2}$ Chloe L. Lash, ${ }^{2,5}$ Kailash L. Dhir, ${ }^{2}$ \\ and Meghan A. Duffy ${ }^{2}$
}

1. Department of Biological Sciences, Purdue University, West Lafayette, Indiana 47907; 2. Department of Ecology and Evolutionary Biology, University of Michigan, Ann Arbor, Michigan 48109; 3. Department of Mathematics and Statistics, Utah State University, Logan, Utah 84322; 4. Natural Resources Ecology Laboratory, Colorado State University, Fort Collins, Colorado 80523; 5. Department of Biology, Valparaiso University, Valparaiso, Indiana 46383

Submitted September 14, 2015; Accepted May 24, 2016; Electronically published September 20, 2016

Online enhancements: appendixes. Dryad data: http://dx.doi.org/10.5061/dryad.cc630.

\begin{abstract}
Aвsтract: Generalist parasites can strongly influence interactions between native and invasive species. Host competence can be used to predict how an invasive species will affect community disease dynamics; the addition of a highly competent, invasive host is predicted to increase disease. However, densities of invasive and native species can also influence the impacts of invasive species on community disease dynamics. We examined whether information on host competence alone could be used to accurately predict the effects of an invasive host on disease in native hosts. We first characterized the relative competence of an invasive species and a native host species to a native parasite. Next, we manipulated species composition in mesocosms and found that host competence results did not accurately predict community dynamics. While the invasive host was more competent than the native, the presence of the native (lower competence) host increased disease in the invasive (higher competence) host. To identify potential mechanisms driving these patterns, we analyzed a two-host, one-parasite model parameterized for our system. Our results demonstrate that patterns of disease were primarily driven by relative population densities, mediated by asymmetry in intra- and interspecific competition. Thus, information on host competence alone may not accurately predict how an invasive species will influence disease in native species.
\end{abstract}

Keywords: biodiversity, Daphnia, dilution effect, invasive species, multihost parasites, pathogen.

\section{Introduction}

Species invasions are a major ecological concern; the influence of parasitism on invasions has recently received sub-

* Corresponding author; e-mail: searlec@purdue.edu.

ORCIDs: Grippi, http://orcid.org/0000-0003-3657-1385; Oleksy, http://orcid.org /0000-0003-2572-5457; Shaw, http://orcid.org/0000-0002-0176-8519; de la Serna, http://orcid.org/0000-0002-6825-8927; Dhir, http://orcid.org/0000-0003-4961-7450.

Am. Nat. 2016. Vol. 188, pp. 554-566. (C) 2016 by The University of Chicago. 0003-0147/2016/18805-56525\$15.00. All rights reserved. DOI: $10.1086 / 688402$ stantial attention. Many invasive species experience enemy release because their natural parasites are absent from invaded communities (Torchin et al. 2003). Alternatively, invasive hosts can carry invasive parasites into their new communities, which can spill over into native species (Garner et al. 2006; Arbetman et al. 2013). In some cases, the presence of a parasite can allow a competitively inferior invasive species to establish in a community (e.g., Borer et al. 2007). However, invasive species may also become infected with native parasites in their new ranges and alter disease dynamics for native hosts (Telfer et al. 2005; Kelly et al. 2009; Mastitsky and Veres 2010). It is this scenario-native parasites infecting invasive hosts - that is the focus of our study.

Invasive species can drive disease amplification or dilution in native hosts. Here, we state that a host species amplifies disease if adding it to a community increases disease in a focal host (measured as the number infected or infection prevalence). A host dilutes disease when the opposite pattern occurs. Invasive species can amplify native parasites (parasite spillback; Kelly et al. 2009) by increasing the abundance of a parasite, leading to apparent competition (Holt and Lawton 1994). This can occur either via increased total host density (Mastitsky and Veres 2010) or, if the invasive species is a highly competent host, due to an increase in overall host competence (Hershberger et al. 2010; Paterson et al. 2013). Alternatively, invasive hosts may reduce infection in the native host (a form of dilution effect; Keesing et al. 2006) through a variety of mechanisms. For example, some hosts that are relatively resistant to infection are able to consume parasites and clear them from the environment (Hall et al. 2009; Venesky et al. 2014). Thus, invasive hosts may amplify or dilute disease for native hosts.

How can we predict the effects of invasive species on native disease? Most predictive approaches focus on the traits or population densities of the invader. The most common 
trait-based approach is to quantify the relative competence of different host species. Highly competent hosts lead to high transmission at the community level, whereas low-competence hosts lead to low rates of transmission (Johnson and Thieltges 2010). All else being equal, adding a highly competent host species to a community should increase disease, while adding a species with low competence should reduce it (LoGiudice et al. 2003; Power and Mitchell 2004; Searle et al. 2011; Johnson et al. 2013). Thus, knowledge of an invasive host's competence may generate predictions of how it will affect native communities.

In addition to host competence, population densities of invasive species can influence whether the invasive species amplifies or dilutes native parasites. The impact of invasive species is predicted to increase at higher densities (Yokomizo et al. 2009), and higher-density species will have a greater ability to amplify or dilute disease (Dobson 2004; Rosa and Pugliese 2007; Hall et al. 2009; Mordecai 2013). Additionally, the impacts of invasive species on native diseases will be influenced by total host density. If invasive individuals replace native individuals such that total host density does not change, the only impacts on disease would be related to host competence. However, if adding a species to a community increases total host density, then the impacts will depend on both the traits of the species and their relative abundance (Rudolf and Antonovics 2005). For example, introducing a low competence host without changes to total host density can cause a dilution effect, while the same host could cause amplification if total host density increases (Mitchell et al. 2002; Power and Mitchell 2004; Roche et al. 2012; Mihaljevic et al. 2014). Amplification and dilution of diseases with density-dependent transmission, where infection rate increases linearly with the density of susceptible individuals (e.g., spore-transmitted parasites), are predicted to depend strongly on whether total host density increases or decreases. In contrast, only dilution is predicted to occur under frequency-dependent transmission, where infection rate increases linearly with the frequency of susceptible hosts (e.g., vector-transmitted parasites; Ruldolf and Antonovics 2005). Patterns of dilution and amplification also depend on how disease risk is measured. For example, Roche et al. (2012) predict that higher host diversity will raise the number of infected individuals by increasing total host density (suggesting amplification) but simultaneously decrease infection prevalence (suggesting dilution). Thus, the impacts of invasive species on native disease dynamics will likely depend on both host competence and host density, as well as the measurement of disease.

In this study, we used experimental and theoretical approaches to explore how disease-related traits and host densities drive disease dynamics. We first performed individuallevel experiments to characterize the host competence of a native and an invasive host species. We then performed a mesocosm experiment to test community-level species interactions and fit a mathematical model to our system to identify potential mechanisms driving the patterns observed in our system.

\section{Study System}

Our native host, Daphnia dentifera Forbes, is common in stratified lakes of North America (Hebert 1995). Our invasive host, Daphnia lumholtzi Sars, is native to Africa, Asia, and Australia (Swar and Fernando 1979; Benzie 1988) and was first discovered in North America in the early 1990s (Havel and Hebert 1993). It has since spread rapidly and is now common throughout much of the United States (Havel and Shurin 2004), where it competes with native species and can alter community structure (Kolar et al. 1997; Johnson and Havel 2001). We refer to D. dentifera and $D$. lumholtzi as the native host and invasive host, respectively. Both species can be reared in isofemale lines in the laboratory (referred to as clones). We used six clones of each species in this study (see table A1 for details; tables A1, B1-B5 available online).

The fungus Metschnikowia bicuspidata (Duffy et al. 2010; Hall et al. 2010) is a common, environmentally transmitted parasite of the native host. When Daphnia ingest spores of this parasite, it penetrates the gut wall and proliferates in the host's hemolymph. It is highly virulent; infected individuals experience reduced fecundity and reduced life span (Ebert et al. 2000; Duffy and Hall 2008). Infections are easily identified because they turn the normally transparent hosts opaque (Duffy and Hall 2008); spores are only released when infected Daphnia die. We used an isolate of M. bicuspidata collected from Baker Lake (Barry County, MI). Isolates from different lakes and years show no detectable variation in infectivity or virulence (Duffy and Sivars-Becker 2007; Searle et al. 2015).

\section{Individual-Level Experiments}

\section{Methods}

We first performed a series of experiments to compare traits of the two host species, including host competence. Quantifying these traits allowed us to make predictions for species interactions and parameterize our model. In each experiment, Daphnia hosts were maintained individually in 50-mL beakers filled with $30 \mathrm{~mL}$ (susceptibility and feeding assays) or $40 \mathrm{~mL}$ (reproduction assay) filtered lake water. All experiments were conducted at $20^{\circ} \mathrm{C}$ with a $16: 8$ photoperiod, and Daphnia were fed daily with $1.0 \times 10^{6}$ cells of a nutritious alga, Ankistrodesmus falcatus. To control for environmental effects, we used second-clutch offspring from third-generation maternal lines unless noted. 
We first quantified reproductive rates in the two host species when provided with ample food. Neonates $(<24 \mathrm{~h}$ old $)$ of six clones from each species were used (see table A1), with 12-15 individuals per clone. We counted offspring three times a week for 35 days, removing offspring after each count. We calculated instantaneous birthrate $(b)$ and intrinsic rate of increase $(r)$ for each clone using methods from Bertram et al. (2013; app. A, apps. A, B available online) and compared between species using Welch's $t$-tests.

We next measured resource acquisition for each species when infected and uninfected. Each individual was a control (unexposed; $n=32-39$ per clone) or exposed to 300 spores $\mathrm{mL}^{-1}$ of the parasite for $24 \mathrm{~h}(n=38-50$ per clone). We measured feeding rates at 8,13 , or 18 days postexposure following the methods of Penczykowski et al. (2014). Each individual was placed in $10 \mathrm{~mL}$ of filtered lake water containing 10,000 cells $A$. falcatus $\mathrm{mL}^{-1}$ for $3 \mathrm{~h}$. We also established ungrazed controls with a range of food concentrations. We measured raw fluorescence using a Trilogy fluorometer (in vivo module; Turner Designs, Sunnyvale, CA) and calculated the number of cells consumed per hour using standard curves from the ungrazed replicates. We also measured each individual's length from the middle of the eye to the base of the tail. The experiment had a destructive sampling design, so each individual was used in only one trial. Individuals that died before their trial or were exposed to the parasite but not infected were excluded from the analyses (for mortality rates, see app. A; fig. A1; figs. A1-A6, B1-B6 available online). We compared body length between species using a Welch's $t$-test and compared feeding rates between species and infection status through time using a linear model.

Our third experiment quantified susceptibility and spore production (two traits that influence host competence) of clones and species. We used four invasive clones and six native clones ( $n=20$ per clone; table A1) from secondgeneration maternal lines. We exposed 7- to 8-day-old Daphnia to 500 parasite spores $\mathrm{mL}^{-1}$ for $22 \mathrm{~h}$. After 16 days, individuals were scored for infection (infected or uninfected); infected individuals were crushed, and parasite spores were quantified with a hemocytometer. We compared infection prevalence between species using a binomial generalized linear model (GLM). We compared spore production among clones and between species using an ANOVA. Individuals that died before the end of the experiment were not included in the analyses.

\section{Results}

The invasive host had greater reproduction (higher $b$ and $r$ ) than the native host $\left(b: t_{9}=3.99, p=.003 ; r: t_{9}=3.63\right.$, $p=.006$; fig. $1 A$ ). Feeding rates did not significantly differ between host species $\left(F_{1,200}=0.88, p=.349\right.$; fig. $\left.1 B\right)$, even though the invasive host was larger (mean length for native $=1,635 \mu \mathrm{m}[\mathrm{SD} \pm 142]$; mean length for invasive $=$ $1,913 \mu \mathrm{m}[\mathrm{SD} \pm 159] ; t_{157}=12.7, p<.001$; fig. A2). There was a significant interaction between infection status and time $\left(F_{1,200}=38.6, p<.001\right)$ where feeding did not differ among treatments 8 days after parasite exposure, but it was reduced in infected individuals of both species at 13 and 18 days (an average $72.2 \%$ reduction at day 18 ; fig. $1 B$ ). The infection assay showed a higher prevalence of infection in the invasive host than in the native host (binomial GLM: $X^{2}(1, N=10)=23.46, p<.001$; fig. $\left.1 C\right)$; average prevalence across clones was 0.23 and 0.81 for the native and invasive hosts, respectively. Spore production at 16 days postexposure did not significantly differ between host species $\left(F_{1,30}=0.23, p=.64\right)$ but varied among clones within spe$\operatorname{cies}\left(F_{7,30}=4.43, p=.002\right.$; fig. $\left.1 D\right)$. Thus, the two host species had similar rates of resource acquisition and parasite production, but the invasive host had faster reproduction and higher parasite susceptibility.

\section{Community-Level Experiment}

\section{Methods}

Based on traits of the two host species (measured in the individual-level experiments), we made predictions for community-level patterns. Because the two hosts had similar rates of resource acquisition but the invasive host had faster reproduction (higher $b$ and $r$ ), we expected the invasive species to be the superior competitor. Additionally, due to the invasive host's higher parasite susceptibility, we predicted that it would have higher infection rates than the native host. We also expected the invasive host to amplify disease in the native host, since adding it to the community would increase average host competence. Conversely, the native host was predicted to dilute disease for the invasive host. Finally, we also expected to see biotic resistance mediated by the parasite (i.e., the native parasite reducing invasive species fitness; Kestrup et al. 2011), where the parasite would reduce population densities of the invasive host more than those of the native host.

To test these predictions, we performed a mesocosm experiment manipulating species composition and parasite presence. The experiment was a $3 \times 2$ factorial design with three species combinations (native only, invasive only, or both species together) and two parasite treatments (exposed or unexposed). This design allowed us to characterize the effects of the invasive host on native hosts, which can occur (1) directly, through resource competition; (2) indirectly, through amplification of a shared parasite; or (3) indirectly, through parasite dilution. Resource competition (1) would be characterized by a negative effect of the invasive host on densities of the native host when the two species are com- 

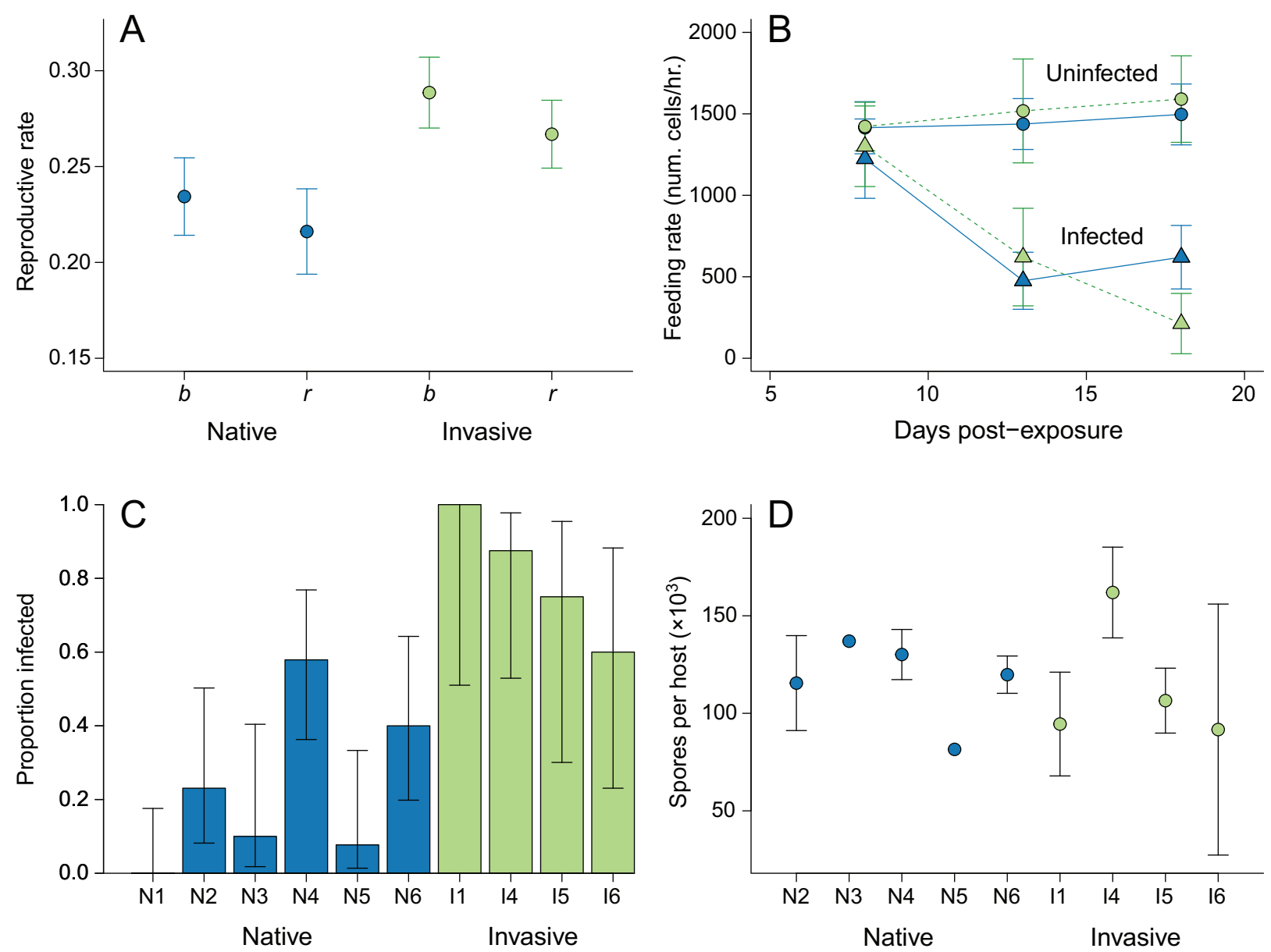

Figure 1: Results from experiments quantifying species traits. $A$, Average instantaneous birthrate $(b)$ and population growth rate $(r)$ are shown for each host species through time, with blue and green circles indicating the native and invasive hosts, respectively. $B$, Feeding rates are indicated for each host species and infection status; circles indicate uninfected individuals, and triangles indicate infected individuals. Results of the susceptibility assay are shown in the bottom panels, with proportion infected $(C)$ and parasite production $(D)$ for the infected individuals. Clones are marked on the $X$-axis $(C, D) ; \mathrm{N}=$ native species; $\mathrm{I}=$ invasive species. Host species differed in rates of reproduction and infection prevalence $(A, C)$ but did not differ in feeding rates or parasite production $(B, D)$. Error bars in $A, B$, and $D$ show \pm 2 SE, while 95\% confidence intervals are shown in $C$.

bined, regardless of parasite presence. Amplification (2) or dilution (3) would be characterized by an increase or decrease in disease, respectively, in the native host when combined with the invasive host (measured as infected host density or infection prevalence).

We conducted this experiment in indoor mesocosms (18.9-L plastic buckets) filled with $15 \mathrm{~L}$ of high-hardness COMBO media (Baer and Goulden 1998), with each treatment combination replicated 10 times. We established a 25:1 nitrogen-to-phosphorus ratio and seeded each mesocosm with $2.5 \times 10^{8}$ cells of the alga Ankistrodesmus falcatus. Average temperatures in the laboratory were $23.2^{\circ} \mathrm{C}(\mathrm{SE} \pm 0.2)$ with a $16 \mathrm{~L}: 8 \mathrm{D}$ photoperiod. For the single-species treatments, we added 45 native or invasive hosts with six genotypes per species (7-8 individuals per genotype). For the mixed-species treatments, we added 35 native hosts and 10 invasive hosts to mimic the early stages of an invasion (also with six genotypes per species). Hosts were acclimated to the mesocosms for 4 days before adding the parasite (at 25 spores $\mathrm{mL}^{-1}$ for the parasite-exposed treatments).

We quantified population densities and infection prevalence every 5 days starting 7 days after initiation of the experiment. On sampling days, each mesocosm was stirred vigorously, a $1-\mathrm{L}$ sample was removed, and $1 \mathrm{~L}$ of fresh COMBO media was added to replace the removed sample. We passed samples through $153-\mu \mathrm{m}$ mesh to concentrate the Daphnia and then counted them under a dissecting microscope. For each individual, we determined species (invasive or native), infection status (infected or uninfected), age (juvenile or adult), and sex (male or female). Mesocosms 
were stirred every 1-2 days throughout the experiment to resuspend algae and the parasite. We supplemented algal and nutrient levels twice a week and added COMBO media as needed to counteract evaporation. The experiment was terminated after 52 days (10 sampling days).

We first calculated integrated population density, integrated infected host density, and infection prevalence (based on integrated densities). Here, integrated density means the area under each curve, which was used to control for temporal variation among replications (see app. A). Since juveniles rarely exhibit visible infections, we calculated infection prevalence using only adults. We performed all analyses two ways: first with separate analyses for each species (two species combinations: alone or combined with the other species) and then with one analysis for the whole community (three species combinations; native only, invasive only, both together). This allowed us to determine the effects of our treatments on single species and the effects on the whole community. We excluded six mesocosms from the analyses (see app. A for justification), with no more than two excluded replicates per treatment. We performed an ANOVA on integrated population density including parasite presence, species combination, and the interaction term as predictors. For integrated infection density and prevalence, we performed an ANOVA comparing species combinations using only parasite-exposed replicates. Significant effects were followed by Tukey's honest significant difference tests. Data collected from the community-level experiment can be found in the Dryad Digital Repository: http://dx.doi.org/10.5061 /dryad.cc630 (Searle et al. 2016).

\section{Results}

We first present our experimental results for each species analyzed separately. Densities of the native host were lower in the presence of the invasive host (an average $45.8 \%$ reduction; $F_{1,31}=25.48, p<.001$ ) and the parasite (an average $30.2 \%$ reduction; $F_{1,31}=9.27, p=.005$; figs. $2 A, \mathrm{~A} 3$ ). Densities of the invasive host were lower in the presence of the native host (an average $27.7 \%$ reduction; $F_{1,15}=$ $6.00, p=.020$ : figs. $2 A$, A3) but, surprisingly, were unaffected by the parasite (a nonsignificant $1.23 \%$ reduction; $F_{1,15}=0.24, p=.630$ ). The interaction between the presence of another species and parasite did not significantly affect density in either species (native: $F_{1,14}=2.87, p=$ .100 ; invasive: $F_{1,15}=.59, p=.446$ ). Infection in the native host was not significantly affected by the presence of the invasive host (integrated infected density: $F_{1,14}=2.70$, $p=.123$; integrated infection prevalence: $F_{1,14}=1.10$, $p=.312$; figs. $2 B, 2 C, A 4)$. In contrast, both infected host density and infection prevalence in the invasive host increased in the presence of the native host (integrated in- fected density: $F_{1,15}=12.60, p=.003$; integrated infection prevalence: $F_{1,15}=11.13, p=.004$; fig. $\left.2 B, 2 C\right)$.

Despite different initial densities of the two host species in the combined treatment, there was no significant difference in densities at the third sampling (17 days later; $t_{15}=$ $1.35, p=.188)$. On this sampling date, average densities for the native and invasive species were $119.1(\mathrm{SD} \pm 70.6)$ and $89.4(\mathrm{SD} \pm 57.3)$ individuals $\mathrm{L}^{-1}$, respectively. The ability of the invasive host to quickly reach similar densities to the native host is likely because the invasive host had faster reproductive rates when provided with ample food (see individual-level experiments). Because of this and because population densities were very low before the third sampling date (fig. A3), differences in integrated densities over the course of the experiment are unlikely to be driven by different initial densities.

The parasites reduced total integrated densities (summing both species when combined) only in the native-only treatment; otherwise, the parasite had little effect on density (a significant parasite $\times$ species combination interaction; $\left.F_{2,31}=3.52, p=.038\right)$. Total densities were high and very similar in the native-only and both-species treatments but were reduced by more than $50 \%$ in the invasive-only treatments (fig. A5). Additionally, species combination affected both the total infected host density and the total infection prevalence (integrated infected density: $F_{2,22}=6.10, p=$ .008; integrated infection prevalence: $F_{2,22}=6.69, p=$ .005; fig. A6). Total infected host density was, on average, $75.0 \%$ and $77.2 \%$ lower in the invasive-only treatments compared to the native-only and both-species treatments, respectively (Tukey's test comparing invasive to both species: $p=.012$; invasive to native species: $p=.027$; native to both species: $p=.934$; fig. A6A). Total integrated infection prevalence showed a similar pattern where the invasiveonly treatment had an average $41.2 \%$ and $55.0 \%$ reduction in prevalence compared to the native-only and both-species treatments, respectively (Tukey's test comparing invasive to both species: $p=.004$; invasive to native species: $p=$ .115 ; native to both species: $p=.312$; fig. A6B). Thus, the invasive-only treatments had the lowest total densities and levels of disease, while the native-only and both-species treatments showed higher total densities and disease.

\section{Mathematical Model}

\section{Methods}

To explore potential mechanisms driving the results in our community-level experiments, we analyzed a two-host, oneparasite model parameterized to our system. The model describes the changes in the densities of susceptible $\left(S_{n}\right)$ and infected $\left(I_{\mathrm{n}}\right)$ native hosts, susceptible $\left(S_{\mathrm{i}}\right)$ and infected $\left(I_{\mathrm{i}}\right)$ invasive hosts, and parasite spores $(P)$ over time. For all 

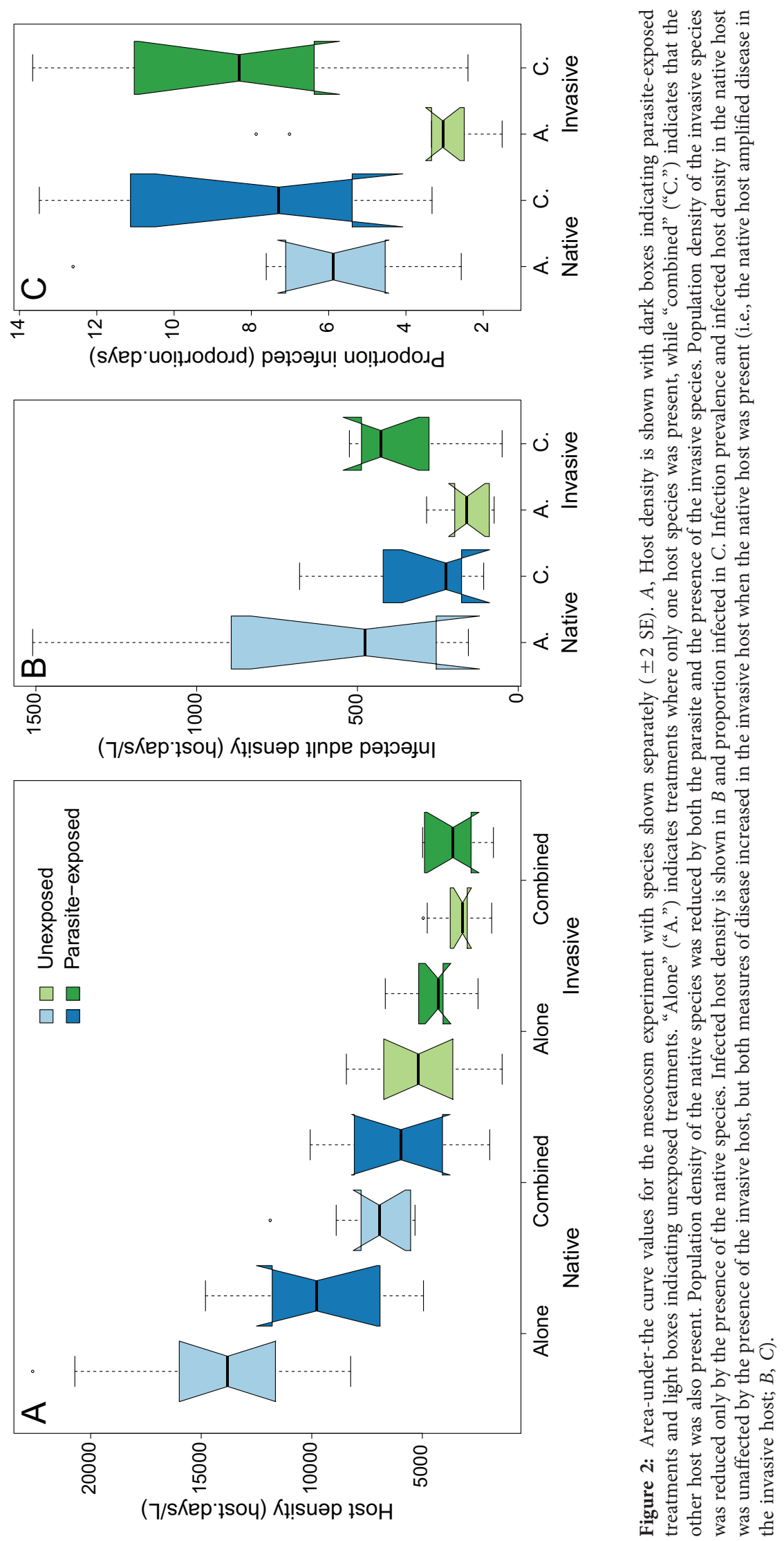

This content downloaded from 129.123.126.245 on November 11, 2016 11:50:59 AM All use subject to University of Chicago Press Terms and Conditions (http://www.journals.uchicago.edu/t-and-c). 
parameters and variables in the model, subscript $\mathrm{n}$ denotes the native host, subscript i denotes the invasive host, and subscript $\mathrm{p}$ denotes the parasite spores. The model is

$$
\begin{aligned}
& \frac{d S_{\mathrm{n}}}{d t}=\overbrace{r_{\mathrm{n}}\left(S_{\mathrm{n}}+\xi I_{\mathrm{n}}\right)\left(1-\frac{S_{\mathrm{n}}+I_{\mathrm{n}}+\alpha_{\mathrm{ni}}\left(S_{\mathrm{i}}+I_{\mathrm{i}}\right)}{K_{\mathrm{n}}}\right)}^{\text {births }}-\overbrace{p_{\mathrm{n}} f_{S_{\mathrm{n}} S_{\mathrm{n}} P}}^{\text {infection }}-\overbrace{\delta S_{\mathrm{n}}}^{\text {sampling }}, \\
& \frac{d I_{\mathrm{n}}}{d t}=\overbrace{p_{\mathrm{n}} f_{S_{\mathrm{n}}} S_{\mathrm{n}} P}^{\text {infection }}-\overbrace{d_{\mathrm{n}} I_{\mathrm{n}}}^{\text {death }}-\overbrace{\delta I_{\mathrm{n}}}^{\text {sampling }}, \\
& \frac{d S_{\mathrm{i}}}{d t}=r_{\mathrm{i}}\left(S_{\mathrm{i}}+\xi I_{\mathrm{i}}\right)\left(1-\frac{S_{\mathrm{i}}+I_{\mathrm{i}}+\alpha_{\mathrm{in}}\left(S_{\mathrm{n}}+I_{\mathrm{n}}\right)}{K_{\mathrm{i}}}\right)-p_{\mathrm{i}} f_{S_{\mathrm{i}}} S_{\mathrm{i}} P-\delta S_{\mathrm{i}}, \\
& \frac{d I_{\mathrm{i}}}{d t}=p_{\mathrm{i}} f_{S_{\mathrm{i}}} S_{\mathrm{i}} P-d_{\mathrm{i}} I_{\mathrm{i}}-\delta I_{\mathrm{i}}, \\
& \frac{d P}{d t}=\overbrace{\beta_{\mathrm{n}} d_{\mathrm{n}} I_{\mathrm{n}}+\beta_{\mathrm{i}} d_{\mathrm{i}} I_{\mathrm{i}}}^{\text {spore release }}-\overbrace{f_{S_{\mathrm{n}}} S_{\mathrm{n}} P-f_{I_{\mathrm{n}}} I_{\mathrm{n}} P-f_{S_{\mathrm{i}} S_{\mathrm{i}} P-f_{I_{\mathrm{i}}} I_{\mathrm{i}} P}^{\text {loss due to uptake }}}^{\text {degradation }}-\overbrace{d_{\mathrm{p}} P}^{\text {sampling }}-\overbrace{\delta P}^{\text {sal }}
\end{aligned}
$$

Table 1 presents the definitions and units for all parameters and variables. In the model, the intra- and interspecific host competition is modeled as Lotka-Volterra competition where $K_{\mathrm{n}}$ and $K_{\mathrm{i}}$ are the species carrying capacities and $\alpha_{\mathrm{n}}$ and $\alpha_{\text {in }}$ are the interspecific competition coefficients. Infected hosts have lower maximum reproductive rates $\left(\xi r_{\mathrm{i}}\right)$ and the same intra- and interspecific competitive effects as susceptible hosts. The host infection rates are proportional to the spore density $(P)$ and the susceptible host ingestion rates $\left(f_{S_{n}}\right.$ and $f_{S_{\mathrm{i}}}$ ) because susceptible hosts become infected when they ingest spores. Spores are released when infected hosts die, and spores are lost due to susceptible and infected host ingestion and degradation. The loss of hosts and spores due to destructive sampling was modeled as a constant removal rate $\delta$.

All parameter values except for the spore degradation rate were estimated from the individual- and communitylevel experiments; for details, see section B1 (secs. B1-B3 in app. B). To compare the model predictions and mesocosm data, we compared the equilibrium densities of the model to long-term density estimates from the mesocosm experiment (hereafter referred to as long-term densities). Because we do not have an estimate for the spore degradation rate, we varied that parameter in the model and identified values where predicted equilibrium densities agreed with qualitative trends in the long-term density estimates. We also performed a limited exploration of parameter space to understand how intraspecific competition ( $K_{\mathrm{i}}$ and $K_{\mathrm{n}}$, a proxy for host population size) and interspecific competition strength $\left(\alpha_{\mathrm{ni}}\right.$ and $\left.\alpha_{\mathrm{in}}\right)$ affected the model predictions. Code for the mathematical model can be found in the Dryad Digital Repository: http:// dx.doi.org/10.5061/dryad.cc630 (Searle et al. 2016). ${ }^{1}$

1. Code that appears in The American Naturalist is provided as a convenience to the readers. It has not necessarily been tested as part of the peer review.
Results

Here, we summarize our theoretical results and their comparisons with the experimental long-term densities. Given the assumptions we made for our model, we do not expect model results to be a perfect quantitative match to those of our empirical results. Instead, we focus on identifying regions of parameter space where the model qualitatively agrees with our experimental findings. Further details about the model analysis and results are presented in sections B2 and B3.

For the estimated intra- and interspecific host competition coefficients, theoretical equilibrium and experimental long-term densities agree qualitatively when the sporedegradation rate is sufficiently low (fig. 3). Specifically, as observed in the mesocosm experiments (fig. $3 A$ ), the model predicts that exposure to a competitor or to the parasite decreases the equilibrium density of both species (fig. $3 C$ table B5). The effect of the competitor on invasive host density was small (fig. $3 C$ ), but the pattern still qualitatively matches the empirical findings (figs. $2 A, 3 A$ ). When the spore degradation rate is sufficiently low (table 1 ), the model predicts that the invasive host dilutes disease for the native host and the native host amplifies disease for the invasive host (fig. 3D; table B5). This prediction agrees qualitatively with our experimental long-term density estimates (cf. fig. $3 B$, $3 D$; table $\mathrm{B} 5$ ). In contrast, when the spore degradation rate is high, the model predicts that both hosts amplify disease for the other host (which was not observed; sec. B2). Thus, our model predicts that low rates of spore degradation are necessary to obtain the observed experimental results.

We estimated intra- and interspecific host competition coefficients by fitting the model to the unexposed mesocosm time series (see sec. B1 for details). For our estimates, $K_{\mathrm{n}}>K_{\mathrm{i}}$ and $\alpha_{\text {in }}<\alpha_{\text {ni }}$, implying that the native hosts are weak competitors and the invasive hosts are strong competitors (both intra- and interspecifically; table 1). Due to their larger carrying capacities, and in agreement with experimental results, native hosts have higher monospecific long-term densities than invasive hosts. Our model predicts that if native and invasive hosts are weak and strong interspecific competitors, respectively, then the invasive host dilutes disease in the native host and the native hosts amplifies disease in the invasive host. This was observed experimentally (fig. $3 B$ ).

When exploring parameter space, we find that increasing a species' interspecific competitive ability makes that species dilute disease more or amplify disease less (fig. $4 A$ ). For example, the invasive host dilutes disease when it is a strong interspecific competitor (large $\alpha_{\text {ni }}$; fig. $4 A$, right side) and amplifies disease when it is a weak interspecific competitor (small $\alpha_{\text {ni }}$; fig. $4 A$, left side). In the absence of interspecific competition $\left(\alpha_{\mathrm{ni}}=\alpha_{\mathrm{in}}=0\right)$, both species amplify disease for the other, agreeing with previous results based on 
Table 1: Variables and parameter definitions and estimates

\begin{tabular}{|c|c|c|c|c|}
\hline Parameter & Definition & Units & Value & $\mathrm{CI}$ or $\mathrm{SE}^{\mathrm{a}}$ \\
\hline$S_{j}$ & Susceptible host density for species $j$ & Individual/L & Variable & $\ldots$ \\
\hline$I_{j}$ & Infected host density for species $j$ & Individual/L & Variable & $\ldots$ \\
\hline$P$ & Spore density & Spore/L & Variable & $\ldots$ \\
\hline$t$ & Time & Day & Variable & $\ldots$ \\
\hline$r_{\mathrm{n}}$ & Native maximum exponential growth rate & Day $^{-1}$ & .206 & $(.154, .274)$ \\
\hline$r_{\mathrm{i}}$ & Invasive maximum exponential growth rate & Day $^{-1}$ & .246 & $(.16, .56)$ \\
\hline$K_{\mathrm{n}}$ & Native carrying capacity & Individual/L & 97.5 & $(29.6,150.0)$ \\
\hline$K_{\mathrm{i}}$ & Invasive carrying capacity & Individual/L & 12.8 & $(2.75,30.9)$ \\
\hline$\alpha_{\mathrm{ni}}$ & Interspecific competition coefficient & Unitless & 2.63 & $(-.28,7.2)$ \\
\hline$\alpha_{\text {in }}$ & Interspecific competition coefficient & Unitless & -.286 & $(-.74, .32)$ \\
\hline$p_{\mathrm{n}}$ & Native probability of infection per spore & $1 /$ spore & $1.45 \times 10^{-5}$ & $\pm 1.39 \times 10^{-5}$ \\
\hline$p_{\mathrm{i}}$ & Invasive probability of infection per spore & $1 /$ spore & $4.87 \times 10^{-5}$ & $\pm 1.03 \times 10^{-5}$ \\
\hline$f_{S_{\mathrm{n}}}$ & Native susceptible host filtering rate & $\mathrm{L}_{\text {day }}{ }^{-1}$ host $^{-1}$ & .0348 & \pm .001 \\
\hline$f_{I_{n}}$ & Native infected host filtering rate & $\mathrm{L}_{\text {day }}{ }^{-1}$ host $^{-1}$ & .0186 & \pm .0095 \\
\hline$f_{S_{i}}$ & Invasive susceptible host filtering rate & $\mathrm{L} \mathrm{day}^{-1}$ host $^{-1}$ & .0361 & \pm .002 \\
\hline$f_{I_{i}}$ & Invasive infected host filtering rate & $\mathrm{L}$ day $^{-1}$ host $^{-1}$ & .0171 & \pm .013 \\
\hline$\beta_{\mathrm{n}}$ & Spores produced per infected native & Spore/individual & 120,000 & $\pm 4,780$ \\
\hline$\beta_{\mathrm{i}}$ & Spores produced per infected invasive & Spore/individual & 124,000 & $\pm 10,853$ \\
\hline$d_{\mathrm{n}}$ & Mortality rate of infected natives & Day $^{-1}$ & .05 & $\ldots$ \\
\hline$d_{\mathrm{i}}$ & Mortality rate of infected invasives & Day $^{-1}$ & .05 & $\ldots$ \\
\hline$\xi$ & Reduction in infected host reproduction & Unitless & .75 & $\ldots$ \\
\hline$\delta$ & Sampling rate & Day $^{-1}$ & .013 & $\ldots$ \\
\hline$d_{\mathrm{P}}$ & Spore degradation rate & Day $^{-1}$ & {$[0, .75]^{\mathrm{b}}$} & $\ldots$ \\
\hline
\end{tabular}

a $95 \%$ confidence interval (CI) or standard error (SE).

${ }^{\mathrm{b}}$ Range of values from model analysis; see section B3 for details. In simulations, we set the spore degradation rate at $d_{\mathrm{P}}=0.5$.

models without interspecific competition (Begon et al. 1992; Begon and Bowers 1994; fig. 4A). We also find that species carrying capacities have a minimal effect on amplification and dilution (fig. $4 \mathrm{~B}$ ) but that large reductions in native population size will cause the invasive host to become an amplifier of disease for the native host (fig. $4 B$, left side). Thus, our model predicts that the invasive host being a stronger intraand interspecific competitor than the native host was an important factor driving the observed experimental results.

Our experimental results using long-term density estimates are very similar to our results using integrated densities (described in the previous section; cf. figs. $2 A, 2 B$ to $3 A$, $3 B$ ). The only qualitative difference is that the invasive host dilutes disease for the native host when analyzing longterm density estimates but not integrated densities (which showed the same trend but not significantly; fig. $2 B$ ). When comparing experimental long-term densities and theoretical equilibrium densities of the model (see above), we focused on the density of infected individuals. However, our results are the same if we instead considered the proportion of infected individuals.

\section{Discussion}

To anticipate the effects of an invasive species on disease dynamics in native hosts, measuring host competence ap- pears to be a useful predictive tool. In our system, the invasive host had higher susceptibility than the native host (fig. 1C), such that adding our invasive host into a native community should increase average host competence (Power and Mitchell 2004; Borer et al. 2007). Therefore, we expected that (1) rates of infection would be higher in the invasive host compared to the native host, (2) the invasive host would amplify disease for the native host, and (3) the parasite would reduce populations of the invasive more than the native host (biotic resistance). These predictions based on host competence did not match the results of our community-level experiments. Instead, we found that (1) rates of infection were higher in the native host than the invasive host, (2) the native host amplified disease in the invasive host, (3) the invasive host either had no effect on native disease or was a diluter (depending on the metric used; figs. $2 B, 2 C, 3 B$ ), and (4) the effects of the parasite on invasive host population density were either neutral or negative (for integrative and long-term densities, respectively), suggesting weak evidence for parasite-driven biotic resistance. These results can be explained, in part, by differences in host population densities, which varied among treatments and mirrored rates of infection (infected host density and infection prevalence). Our theoretical work suggests that asymmetric host competition was a driving mechanism of the density and disease patterns we observed. Overall, the effects of invasive hosts 

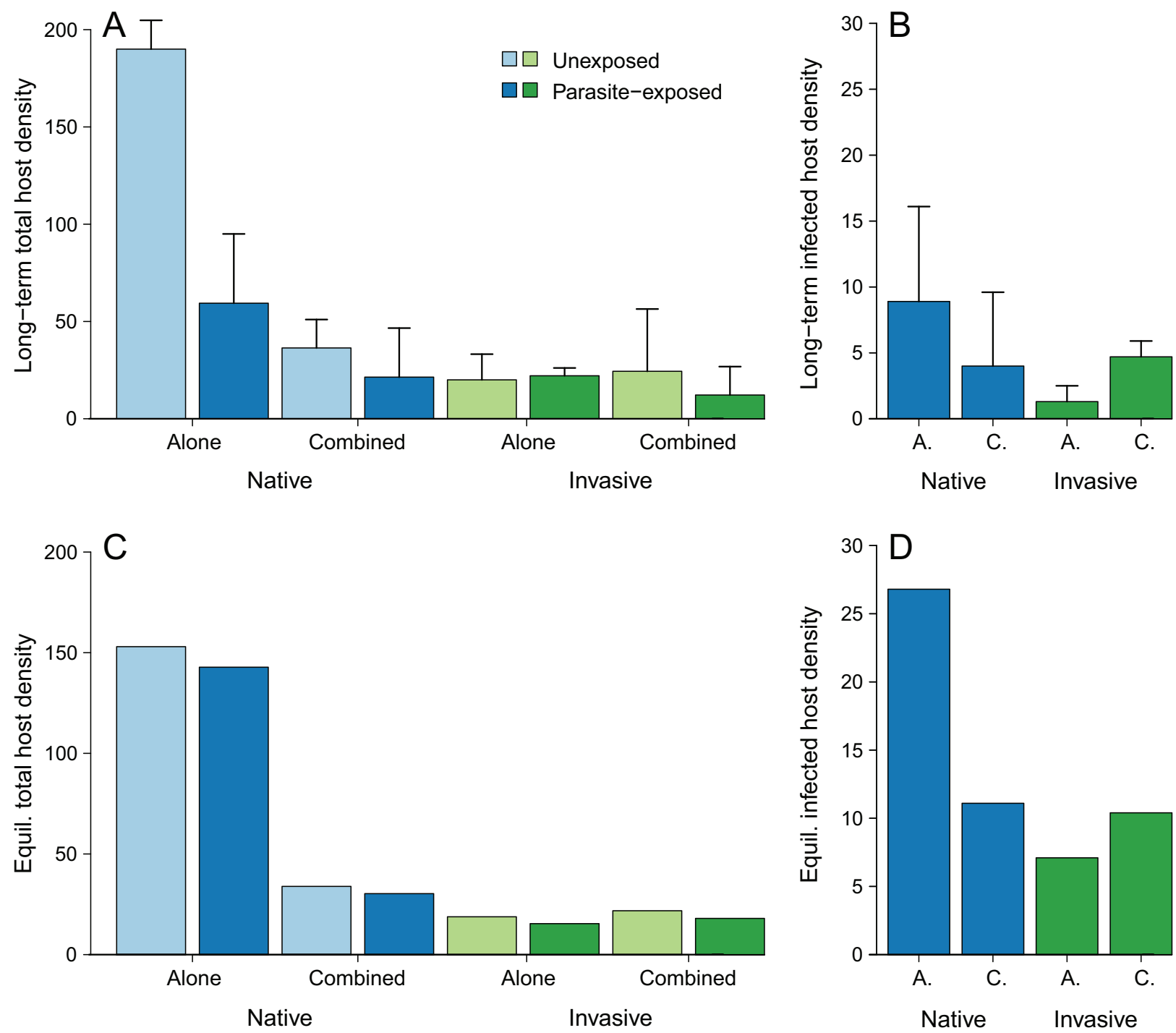

Figure 3: Comparison of empirical and modeling results. Long-term density estimates from the mesocosms are shown for total population density $(A)$ and infected host density $(B)$. Equilibrium densities from the model are shown for total population density $(C)$ and infected host density $(D)$. Dark bars indicate parasite-exposed treatments, and light bars are unexposed treatments. "Alone" ("A.") indicates treatments where only one host species was present, while "combined" ("C.") indicates that the other host was also present. Error bars represent \pm 2 SD. Overall, there was good qualitative agreement between our long-term density estimates from the mesocosm and equilibrium estimates from the model (cf. $A, B$ to $C, D$ ).

on native disease may depend on a wide range of host attributes, including disease-related host traits (e.g., host competence) and population-level characteristics (e.g., population density and competitive ability), as well as attributes of the parasite (e.g., degradation rate in the environment). Host population density may be of equal or greater importance to community disease dynamics than relative host competence (Mordecai 2013; Mihaljevic et al. 2014; Wojdak et al. 2014; this study).

Our model predicts that asymmetric competition, specifically the invasive host being a stronger intra- and inter- specific competitor than the native host, drove the patterns of amplification and dilution we observed. This asymmetry drives the observed dynamics in the following way. Individual hosts become infected via contact with spores, whose density is determined by average host competence and total host density. When invasive hosts are added to a system with native hosts, average host competence increases. However, because invasive hosts are strong interspecific competitors, total host density decreases markedly. The decrease in total host density overwhelms the increase in average host competence, causing infected native host density and spore 

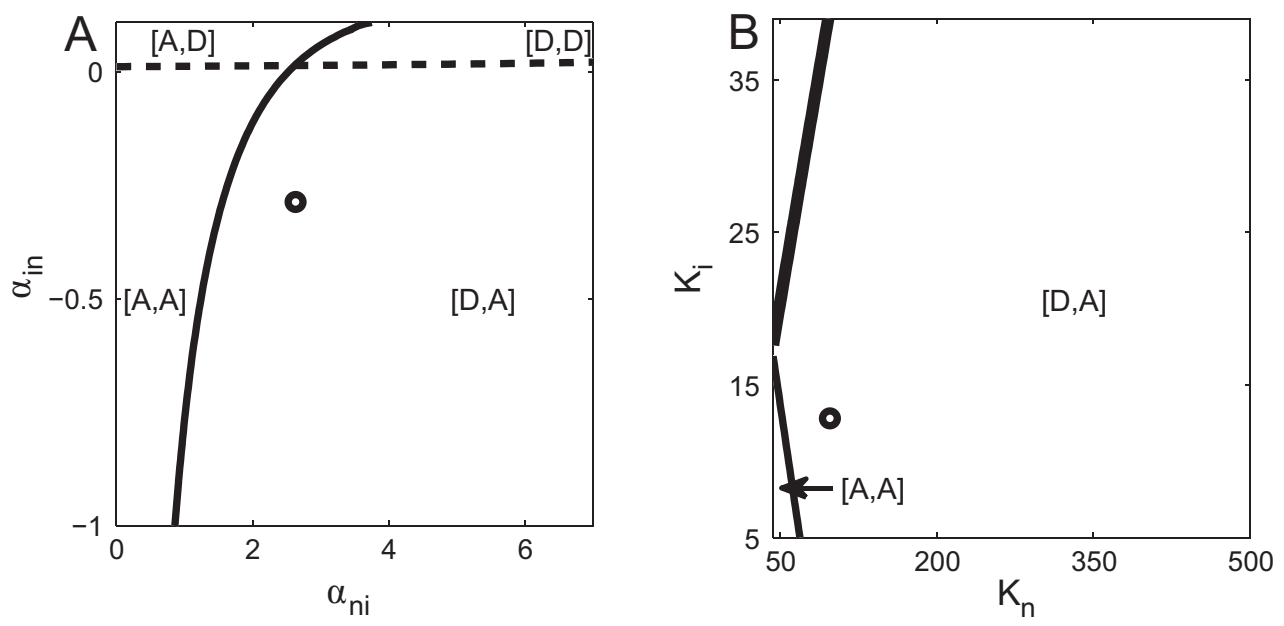

Figure 4: Dependence of dilution and amplification on host interspecific competition coefficients $(A)$ and host carrying capacities $(B)$. For each region of parameter space, the first letter in brackets corresponds to whether the invasive species dilutes (D) or amplifies (A) disease in the native host; the second letter denotes whether the native host dilutes or amplifies disease in the invasive host. The thin solid (thin dashed) curve denotes parameters at which the invasive (native) host switches from diluting to amplifying, or vice versa. Above the thick solid black line in $B$, the host-host-parasite endemic coexistence equilibrium does not exist. The black circles denote the locations of the parameter values in table 1.

density to decrease (i.e., the native host experiences a dilution effect). In contrast, adding the native hosts to a system with invasive hosts reduces average host competence but greatly increases total host density. The increase in total host density overwhelms the decrease in average host competence, causing spore density to increase. Consequently, infected invasive host density increases (i.e., the invasive host experiences amplification). Note that our estimate of $\alpha_{\text {in }}$ is negative, implying that native hosts have a positive effect on the growth rate of invasive hosts. The sign of the estimate is unexpected and likely due to difficulty associated with estimating the parameter from transient dynamics (95\% confidence interval spans -0.74 to 0.32 ; see secs. B1, B3 for more discussion). However, if we set $\alpha_{\text {in }}$ to zero or a small positive value (implying that the native host is a weak interspecific competitor), our qualitative results regarding amplification and dilution do not change (secs. B2, B3).

How likely is it that invasive hosts are stronger competitors than native hosts, as our modeling suggests? The invasive host had higher rates of reproduction than the native host (fig. 1A) but, nevertheless, had lower population densities (figs. $2 A, \mathrm{~A} 3$ ), suggesting stronger intraspecific competition. In fact, two mesocosms were excluded from our analyses because the invasive host went extinct or nearly extinct. These differences were unlikely due to different rates of resource acquisition since feeding rates did not differ between host species (fig. 1B). Instead, the low densities of the invasive host were likely due to production of males. Daphnia produce female asexual offspring during favorable conditions but can asexually produce male offspring in response to crowding, temperature, photoperiod, or exposure to hormones (Stross and Hill 1965; Lampert et al. 2012). Production of males reduces population growth because males cannot reproduce on their own and, after sexual reproduction, females produce diapausing eggs (ephippia), which take months or years to hatch (Cáceres 1998). In our mesocosm experiment, all but one invasive-only replicate had $>30 \%$ males in the population at some time point (average maximum percent male $=40.6 \%[\mathrm{SD}=6.20]$ ), while none of the native populations reached a $5 \%$ male population (average maximum percent male $=1.11 \%$ [SD = 1.09]). We do not know whether this pattern is also found in nature or whether it is due to the conditions in our mesocosms. However, a tendency to reproduce sexually is likely linked to Daphnia lumholtzi's invasiveness, as dispersal occurs via sexually produced eggs (Havel and Shurin 2004). The propensity for the invasive host to produce males results in lower monospecific long-term densities of invasive hosts compared to monospecific long-term densities of native hosts, which is captured by higher intraspecific competition between invasive hosts in our model. Native host population densities are more strongly affected by the presence of the invasive host than vice versa (cf. the difference between native alone and combined without disease to the difference between invasive alone and combined without disease; fig. $3 A$ ). This suggests that invasive hosts are also stronger interspecific competitors than native hosts. If we allowed invasive intraspecific competition to be low, our model predicts increased density of invasive hosts and a dilution effect when the native species was added (which was not observed). Thus, our experimental and theoretical results indicate that asymmetrical competition, partly driven by the propensity of in- 
vasive hosts to invest in sexual reproduction, drove disease dynamics.

The spore degradation rate, a parameter for which we do not have an estimate, also has a strong influence on model dynamics. Qualitative agreement between the model and the data occurs only for the estimated parameter values when the spore degradation rate is sufficiently small $\left(d_{\mathrm{p}}<0.75\right)$. If the spore degradation rate is higher (e.g., due to ultraviolet radiation; Overholt et al. 2012), the model predicts that both hosts amplify disease (fig. B4). Thus, our model predicts that spore degradation does not occur quickly in our system. However, it is important to note that the range of $d_{\mathrm{p}}$ values for which amplification and dilution occur strongly depend on the values of $\alpha_{\mathrm{in}}, \alpha_{\mathrm{ni}}, K_{\mathrm{n}}$, and $K_{\mathrm{i}}$. For example, for parameter values similar but not identical to those in table 1 , qualitative agreement between the model and the data occurs only when spore degradation rate is sufficiently large $\left(d_{\mathrm{p}}>1.1\right.$; sec. B3). Thus, there is a need for further investigation into how host inter- and intraspecific competition affect dilution and amplification and how these effects depend on other parasite-related traits (e.g., spore ingestion rates and spore degradation rates).

One key difference between our model and previous theoretical work is that our model includes interspecific host competition. Previous theoretical work on environmentally transmitted parasites without interspecific host competition predicts that both species will amplify disease (Begon and Bowers 1994) because the addition of either species increases total host density. In our model, due to the interspecific host competition, the total host density when both species coexist is intermediate of the host densities of the monospecific systems. This change in density results in the invasive host diluting rather than amplifying the disease for the native host. Hence, a key finding of our study is that interspecific competition can be an important mechanism driving patterns of dilution or amplification in systems with environmentally transmitted parasites.

While we have focused on infected host density in our model, dilution and amplification have also been discussed in the context of disease prevalence (proportion of hosts infected; Keesing et al. 2006). When analyzed in terms of disease prevalence, our theoretical and experimental results still show that dilution and amplification of disease cannot be predicted from host competence alone. Specifically, we find that the addition of the invasive species can increase long-term estimates of native disease prevalence in the data $(0.13$ [SE $=0.01]$ to $0.16[\mathrm{SE}=0.02])$ and the model $(0.09$ to 0.22 ) as predicted by the competence of the invasive host. However, we also observe that the native species increases long-term estimates of invasive host prevalence in the data $(0.06[\mathrm{SE}=0.02]$ to $0.28[\mathrm{SE}=0.10])$ and the model $(0.29$ to 0.50 ), which is not predicted by native host competence. Thus, our empirical and theoretical results show that between- species interactions can alter amplification and dilution of disease regardless of how it is measured.

A number of theoretical studies have investigated how shared parasites affect coexistence between host species, with parasite-mediated coexistence or exclusion being some of the possible outcomes (e.g., Begon et al. 1992; Begon and Bowers 1994; Dobson 2004; Rudolf and Antonovics 2005). Due to the differences in interspecific competitive ability, one might expect that the native and invasive host cannot exist in the absence of the parasite. However, because the invasive host is both a strong inter- and intraspecific competitor, coexistence is possible. In particular, for the Lotka-Volterra competition portion of our model, the product of intraspecific competition coefficients is greater than the product of the interspecific competition coefficients, which is the condition for stable coexistence. Thus, coexistence of the two host species was possible due to a combination of strong resource competition (asymmetric carrying capacities) and strong interference competition (asymmetric $\alpha$ values; Persson 1985). Note that our model predicts that coexistence between two hosts is possible with or without the parasite, suggesting that coexistence is not mediated by the parasite.

Previous theoretical work without interspecific host competition (Rudolf and Antonovics 2005) has argued that density-dependent and frequency-dependent transmission affect dilution and amplification differently. Under densitydependent transmission, dilution is expected when host density changes are substitutive (i.e., total host density does not change when new hosts are added) and amplification is expected when host density changes are additive (i.e., total host density increases when new hosts are added). In contrast, dilution is always predicted to occur under frequency-dependent transmission (Rudolf and Antonovics 2005). There is very little theory about how interspecific host competition should change these patterns (but see Strauss et al. 2015). Sporebased transmission is expected to be a density-dependent process because transmission rates increase linearly with infectious propagule density. In contrast, vector-based transmission is expected to be a frequency-dependent process because the rate of transmission depends on the frequency of infected vectors. The observed patterns of amplification and dilution for our spore-transmitted parasite do not align with either set of predictions. The two likely causes for this are that interspecific competition is present and strong in our system and that both susceptible and infected hosts clear spores from the environment (spore ingestion). Indeed, when both of these components are removed from the model, the model dynamics agree with predictions for density-dependent transmission processes. Thus, the mode of transmission of a parasite will likely influence how the addition of a species will influence disease.

Understanding how invasive species interact with native hosts and parasites is essential as species invasions and ep- 
idemics become an increasing concern. We found that the impacts of invasive species on native communities cannot be predicted based on host competence alone. Instead, host population density and competitive asymmetries, characteristics that are not directly linked with disease dynamics, shaped the relationships between an invasive host species, a native host species, and a native parasite. In fact, disease dynamics in a number of natural communities can be driven by factors other than host competence (Ogden and Tsao 2009; Roche et al. 2012; Mordecai 2013). Similar patterns may be found with the addition of any host species to a community, whether invasive or native. Thus, caution is warranted when using host competence alone to predict how invasive species might alter the dynamics of native hosts and their parasites.

\section{Acknowledgments}

We would like to thank R. W. Bilich, A. M. Bromilow, and M. R. Christie for their assistance, S. R. Hall for providing code to calculate reproductive rates, and R. M. Penczykowski for logistical advice. We also thank the anonymous reviewers, who provided valuable feedback on our manuscript. This project was funded by a National Science Foundation CAREER grant to M.A.D. (DEB-1305836).

\section{Literature Cited}

Arbetman, M. P., I. Meeus, C. L. Morales, M. A. Aizen, and G. Smagghe. 2013. Alien parasite hitchhikes to Patagonia on invasive bumblebee. Biological Invasions 15:489-494.

Baer, K. N., and C. E. Goulden. 1998. Evaluation of a high-hardness COMBO medium and frozen algae for Daphnia magna. Ecotoxicology and Environmental Safety 39:201-306.

Begon, M., and R. G. Bowers. 1994. Host-host-pathogen models and microbial pest control: the effect of host self-regulation. Journal of Theoretical Biology 169:275-287.

Begon, M., R. G. Bowers, N. Kadianakis, and D. E. Hodgkinson. 1992. Disease and community structure: the importance of host selfregulation in a host-host-pathogen model. American Naturalist 139: $1131-1150$.

Benzie, J. A. 1988. The systematics of Australian Daphnia (Cladocera: Daphniidae): species descriptions and keys. Hydrobiologia 166: 95-161.

Bertram, C. R., M. Pinkowski, S. R. Hall, M. A. Duffy, and C. E. Cáceres. 2013. Trait-mediated indirect effects, predators, and disease: test of a size-based model. Oecologia (Berlin) 173:1023-1032.

Borer, E. T., P. R. Hosseini, E. W. Seabloom, and A. P. Dobson. 2007. Pathogen-induced reversal of native dominance in a grassland community. Proceedings of the National Academv of Sciences of the USA 104:5473-5478.

Cáceres, C. 1998. Interspecific variation in the abundance, production, and emergence of Daphnia diapausing eggs. Ecology 79:1699-1710.

Dobson, A. 2004. Population dynamics of pathogens with multiple host species. American Naturalist 164(suppl.):S64-S78.

Duffy, M. A., C. E. Cáceres, S. R. Hall, A. J. Tessier, and A. R. Ives. 2010. Temporal, spatial, and between-host comparisons of patterns of parasitism in lake zooplankton. Ecology 91:3322-3331.
Duffy, M. A., and S. R. Hall. 2008. Selective predation and rapid evolution can jointly dampen effects of virulent parasites on Daphnia populations. American Naturalist 171:499-510.

Duffy, M. A., and L. Sivars-Becker. 2007. Rapid evolution and ecological host-parasite dynamics. Ecology Letters 10:44-53.

Ebert, D., M. Lipsitch, and K. L. Mangin. 2000. The effect of parasites on host population density and extinction: experimental epidemiology with Daphnia and six microparasites. American Naturalist 156: 459-477.

Garner, T. W., M. W. Perkins, P. Govindarajulu, D. Seglie, S. Walker, A. A. Cunningham, and M. C. Fisher. 2006. The emerging amphibian pathogen Batrachochytrium dendrobatidis globally infects introduced populations of the North American bullfrog, Rana catesbeiana. Biology Letters 2:455-459.

Hall, S. R., C. R. Becker, J. L. Simonis, M. A. Duffy, A. J. Tessier, and C. E. Cáceres. 2009. Friendly competition: evidence for a dilution effect among competitors in a planktonic host-parasite system. Ecology 90:791-801.

Hall, S. R., R. Smyth, C. R. Becker, M. A. Duffy, C. J. Knight, S. MacIntyre, A. J. Tessier, and C. E. Cáceres. 2010. Why are Daphnia in some lakes sicker? disease ecology, habitat structure, and the plankton. BioScience 60:363-375.

Havel, J. E., and P. D. N. Hebert. 1993. Daphnia lumholtzi in North America: another exotic zooplankter. Limnology and Oceanography 38:1823-1827.

Havel, J. E., and J. B. Shurin. 2004. Mechanisms, effects, and scales of dispersal in freshwater zooplankton. Limnology and Oceanography 49:1229-1238.

Hebert, P. 1995. The Daphnia of North America: an illustrated fauna. CyberNatural Software, University of Guelph.

Hershberger, P. K., B. K. Leeuw, J. L. Gregg, C. A. Grady, K. M. Lujan, S. K. Gutenberger, M. K. Purcell, J. C. Woodson, J. R. Winton, and M. J. Parsley. 2010. Amplification and transport of an endemic fish disease by an introduced species. Biological Invasions 12:3665-3675.

Holt, R., and J. Lawton. 1994. The ecological consequences of shared natural enemies. Annual Review of Ecology, Evolution, and Systematics 25:495-520.

Johnson, J. L., and J. E. Havel. 2001. Competition between native and exotic Daphnia: in situ experiments. Journal of Plankton Research 23:373-387.

Johnson, P. T. J., D. L. Preston, J. T. Hoverman, and K. L. Richgels. 2013. Biodiversity decreases disease through predictable changes in host community competence. Nature 494:230-233.

Johnson, P. T. J., and D. W. Thieltges. 2010. Diversity, decoys and the dilution effect: how ecological communities affect disease risk. Journal of Experimental Biology 213:961-970.

Keesing, F., R. D. Holt, and R. S. Ostfeld. 2006. Effects of species diversity on disease risk. Ecology Letters 9:485-498.

Kelly, D. W., R. A. Paterson, C. R. Townsend, R. Poulin, and D. M. Tompkins. 2009. Parasite spillback: a neglected concept in invasion ecology? Ecology 90:2047-2056.

Kestrup, Å. M., S. H. Thomas, K. van Rensburg, A. Ricciardi, and M. A. Duffy. 2011. Differential infection of exotic and native freshwater amphipods by a parasitic water mold in the St. Lawrence River. Biological Invasions 13:769-779.

Kolar, C. S., J. C. Boase, D. F. Clapp, and D. H. Wahl. 1997. Potential effect of invasion by an exotic zooplankter, Daphnia lumholtzi. Journal of Freshwater Ecology 12:521-530.

Lampert, W., K. P. Lampert, and P. Larsson. 2012. Induction of male production in clones of Daphnia pulex by the juvenoid hormone 
methyl farnesoate under short photoperiod. Comparative Biochemistry and Physiology C: Toxicology and Pharmacology 156:130-133.

LoGiudice, K., R. S. Ostfeld, K. A. Schmidt, and F. Keesing. 2003. The ecology of infectious disease: effects of host diversity and community composition on Lyme disease risk. Proceedings of the National Academy of Sciences of the USA 100:567-571.

Mastitsky, S. E., and J. K. Veres. 2010. Field evidence for a parasite spillback caused by exotic mollusc Dreissena polymorpha in an invaded lake. Parasitology Research 106:667-675.

Mihaljevic, J. R., M. B. Joseph, S. A. Orlofske, and S. H. Paull. 2014. The scaling of host density with richness affects the direction, shape, and detectability of diversity-disease relationships. PLoS ONE 9:e97812.

Mitchell, C. E., D. Tilman, and J. V. Groth. 2002. Effects of grassland plant species diversity, abundance, and composition on foliar fungal disease. Ecology 83:1713-1726.

Mordecai, E. A. 2013. Despite spillover, a shared pathogen promotes native plant persistence in a cheatgrass-invaded grassland. Ecology 94:2744-2753.

Ogden, N. H., and J. L. Tsao. 2009. Biodiversity and Lyme disease: dilution or amplification? Epidemics 1:196-206.

Overholt, E. P., S. R. Hall, C. E. Williamson, C. K. Meikle, M. A. Duffy, and C. E. Cáceres. 2012. Solar radiation decreases parasitism in Daphnia. Ecology Letters 15:47-54.

Paterson, R. A., C. A. Rauque, M. V. Fernandez, C. R. Townsend, R. Poulin, and D. M. Tompkins. 2013. Native fish avoid parasite spillback from multiple exotic hosts: consequences of host density and parasite competency. Biological Invasions 15:2205-2218.

Penczykowski, R. M., B. C. P. Lemanski, R. D. Sieg, S. R. Hall, J. H. Ochs, J. Kubanek, and M. A. Duffy. 2014. Poor resource quality lowers transmission potential by changing foraging behaviour. Functional Ecology 28:1245-1255.

Persson, L. 1985. Asymmetrical competition: are larger animals competitively superior? American Naturalist 126:261-266.

Power, A. G., and C. E. Mitchell. 2004. Pathogen spillover in disease epidemics. American Naturalist 164(suppl.):S79-S89.

Roche, B., A. P. Dobson, J. F. Guégan, and P. Rohani. 2012. Linking community and disease ecology: the impact of biodiversity on pathogen transmission. Philosophical Transactions of the Roval Society B 367:2807-2813.

Rosa, R., and A. Pugliese. 2007. Effects of tick population dynamics and host densities on the persistence of tick-borne infections. Mathematical Biosciences 208:216-240.

Rudolf, V. H., and J. Antonovics. 2005. Species coexistence and pathogens with frequency-dependent transmission. American Naturalist 166:112-118.
Searle, C. L., L. M. Biga, J. W. Spatafora, and A. R. Blaustein. 2011. A dilution effect in the emerging amphibian pathogen Batrachochytrium dendrobatidis. Proceedings of the National Academy of Science 108: 16322-16326.

Searle, C. L., M. H. Cortez, K. K. Hunsberger, D. C. Grippi, I. A. Oleksy, C. L. Shaw, S. B. de la Serna, C. L. Lash, K. L. Dhir, and M. A. Duffy. 2016. Data from: Population density, not host competence, drives patterns of disease in an invaded community. American Naturalist, Dryad Digital Repository, http://dx.doi.org/10.5061/dryad.cc630.

Searle, C. L., J. H. Ochs, C. E. Cáceres, S. Chiang, N. M. Gerardo, S. R. Hall, and M. A. Duffy. 2015. Plasticity, not genetic variation, drives infection success of a fungal parasite. Parasitology 142:839-848.

Strauss, A. T., D. J. Civitello, C. E. Cáceres, and S. R. Hall. 2015. Success, failure, and ambiguity of the dilution effect among competitors. Ecology Letters 18:916-926.

Stross, R., and J. Hill. 1965. Diapause induction in Daphnia requires two stimuli. Science 150:1462-1464.

Swar, D. B., and C. H. Fernando. 1979. Seasonality and fecundity of Daphnia lumholtzi Sars in Lake Phewa, Nepal. Hydrobiologia 64:261-268.

Telfer, S., K. J. Bown, R. Sekules, M. Begon, T. Hayden, and R. Birtles. 2005. Disruption of a host-parasite system following the introduction of an exotic host species. Parasitology 130:661-668.

Torchin, M. E., K. D. Lafferty, A. P. Dobson, V. J. McKenzie, and A. M. Kuris. 2003. Introduced species and their missing parasites. Nature 421:628-630.

Venesky, M. D., X. Liu, E. L. Sauer, and J. R. Rohr. 2014. Linking manipulative experiments to field data to test the dilution effect. Journal of Animal Ecology 83:557-565.

Wojdak, J. M., R. M. Edman, J. A. Wyderko, S. A. Zemmer, and L. K. Belden. 2014. Host density and competency determine the effects of host diversity on trematode parasite infection. PLoS ONE 9:e105059.

Yokomizo, H., H. P. Possingham, M. B. Thomas, and Y. M. Buckley. 2009. Managing the impact of invasive species: the value of knowing the density-impact curve. Ecological Applications 19:376-386.

\section{References Cited Only in the Online Appendixes}

Abrams, P. A. 2009. When does greater mortality increase population size? the long history and diverse mechanisms underlying the hydra effect. Ecology Letters 12:462-474.

Associate Editor: Benjamin M. Bolker Editor: Judith L. Bronstein 